\title{
DIE GRIECHISCHEN
}

\section{CHRISTLICHEN SCHRIFTSTELLER \\ DER}

\section{ERSTEN DREI JAHRHUNDERTE}

HERAUSGEGEBEN VON DER KIRCHENVÄTER-COMMISSION

DER PREUSSISCHEN AKADEMIE DER WISSENSCHAFTEN

\author{
न्वृन्त \\ ORIGENES \\ SIEBENTER BAND
}

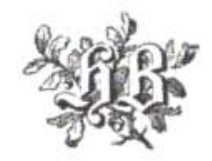

LEIPZIG

J. C. HINRICHS'sche BUCHHANDLUNG 1921 
IN DER REIHENFOLGE DES ERSCHEINENS BAND 30

Druck von Aug ast Pries in Leipzig. 


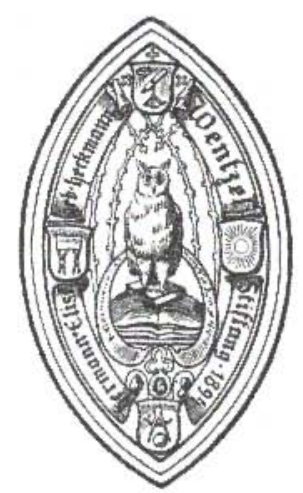

Hermann und Elise geb. Heckmann WENTZEL-STIFTUNG 



\title{
ORIGENES WERKE
}

SIEBENTER BAND

\section{HOMILIE N ZUM HEXATEUCH \\ IN RUFINS ÜBERSETZUNG}

\author{
HERADSGEGEBEN
}

IM AUFTRAGE DER KIRCHENVATER-COMMISSION

DER PREUSSISOHEN AKADEMIE DER WISSENSCHAFTEN

VON

Prof. DR. W. A. BAEHRENS

ZWEITER 'TEIL

DIE HOMILIEN ZU NUMERI, JOSUA UND JUDICES

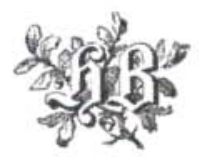

LEIPZIG

.J. C. HINRICHS'sche BUCHHANDLUNG 
